\title{
Papers
}

\section{Extracorporeal shock wave therapy for plantar fasciitis: randomised controlled multicentre trial}

\author{
Michael Haake, Mathias Buch, Carsten Schoellner, Felix Goebel, Martin Vogel, Ingo Mueller, \\ Jörg Hausdorf, Karin Zamzow, Carmen Schade-Brittinger, Hans-Helge Mueller
}

\begin{abstract}
Objective To determine the effectiveness of extracorporeal shock wave therapy compared with placebo in the treatment of chronic plantar fasciitis. Design Randomised, blinded, multicentre trial with parallel group design.

Setting Nine hospitals and one outpatient clinic in Germany.

Participants 272 patients with chronic plantar fasciitis recalcitrant to conservative therapy for at least six months: 135 patients were allocated extracorporeal shock wave therapy and 137 were allocated placebo. Main outcome measures Primary end point was the success rate 12 weeks after intervention based on the Roles and Maudsley score. Secondary end points encompassed subjective pain ratings and walking ability up to a year after the last intervention.

Results The primary end point could be assessed in $94 \%(\mathrm{n}=256)$ of patients. The success rate 12 weeks after intervention was $34 \%(n=43)$ in the extracorporeal shock wave therapy group and 30\% $(\mathrm{n}=39)$ in the placebo group $(95 \%$ confidence interval $-8.0 \%$ to $15.1 \%)$. No difference was found in the secondary end points. Few side effects were reported. Conclusions Extracorporeal shock wave therapy is ineffective in the treatment of chronic plantar fasciitis.
\end{abstract}

\section{Introduction}

Plantar fasciitis is a common cause of heel pain, affecting $10 \%$ of the general population. ${ }^{1}$ It may be due to injury at the origin of the plantar fascia or to biomechanical abnormalities of the foot. ${ }^{23} \mathrm{~A}$ heel spur may be present, but has also been reported in up to $27 \%$ of patients without symptoms. ${ }^{1}$

Standard treatment for plantar fasciitis is conservative, but about $10 \%$ of patients fail to respond. ${ }^{4}$ Surgery is recommended eventually, but is unsuccessful in $2 \%$ to $35 \%$ of patients. ${ }^{5}$ For both conservative and surgical methods there is only limited evidence for a short term reduction of pain from local treatment with corticosteroids. $^{6}$

Extracorporeal shock wave therapy is well established for the treatment of urolithiasis. ${ }^{7}$ It was introduced in the early 1990s for the treatment of insertion tendinopathies. ${ }^{8}$ Extracorporeal shock wave therapy for orthopaedic diseases is thought to provide long lasting analgesia and stimulate the healing process. It has been recommended as treatment for chronic plantar fasciitis in patients unresponsive to conservative treatment. ${ }^{9-13}$ Two shock wave devices are currently approved by the Food and Drug Administration. ${ }^{12} 13$

The efficacy of extracorporeal shock wave therapy in plantar fasciitis cannot be ascertained owing to the poor quality of methods in previous studies. ${ }^{14} \mathrm{We}$ aimed to determine its effectiveness in chronic plantar fasciitis.

\section{Materials and methods}

Our study was a randomised, blinded, multicentre trial with a two sample parallel group design. Central randomisation and independent monitoring was conducted according to the Committee for Proprietary Medicinal Products and International Conference on Harmonisation guidelines for good clinical practice and statistical principles in clinical trials.

Patients were recruited in seven university hospitals, two clinics, and one practice in Germany (see bmj.com for inclusion and exclusion criteria). In cases where the clinical diagnosis was uncertain, the centres were advised to perform additional diagnostic tests, such as electromyography. Signed informed consent was obtained from all patients before randomisation.

Patients were randomised to receive either extracorporeal shock wave therapy (135 patients) or placebo (137 patients). The study doctor was told by telephone what treatment had been allocated to his or her patient when the patient turned up for the first intervention. Random permuted blocks of sizes six and four were used to provide each centre with a separate computer generated list of random treatment assignments.

\section{Intervention}

Extracorporeal shock wave therapy was provided by a Dornier Epos Ultra lithotripter (Dornier Medizintechnik, Wessling, Germany) equipped with an outline 7.5 $\mathrm{MHz}$ linear array ultrasound positioning system. Before recruitment of patients, the study doctors were given training in the diagnosis of plantar fasciitis and treatment with extracorporeal shock wave therapy or placebo.

Extracorporeal shock wave therapy comprised 4000 impulses of a positive energy flux density $(0.08$ $\mathrm{mJ} / \mathrm{mm}^{2}$ ) under local anaesthesia with $2 \mathrm{ml}$ mepi-
Orthopädische Klinik, Universität Regensburg, 93077 Bad Abbach, Germany

Michael Haake associate assistant professor

Orthopädische Klinik Kassel, 43131 Kassel, Germany Mathias Buch assistant medical director

Orthopädische Klinik, Johannes Gutenberg

Universität Mainz 55131 Mainz, Germany Carsten Schoellner senior resident

Klinik für

Orthopädie, Martin Luther Universität

Halle, 06097 Halle, Germany

Felix Goebel senior resident

Rehbergklinik St Andreasberg, 37444 St. Andreasberg, Germany

Martin Vogel

Orthopädische Klinik,

Christian-AlbrechtsUniversität Kiel, 24105 Kiel,

Germany

Ingo Mueller senior resident

continued over

bmj.com 2003;327:75

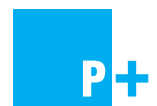

Tables and criteria for inclusion or exclusion of patients appear on bmj.com 
Orthopädische Klinik, Ludwig Maximilians Universität

München, 81377

München, Germany

Jörg Hausdorf

senior registrar

Medizinische

Biometrie und

Epidemiologie,

Philipps-Universität

Marburg, 35033

Marburg, Germany

Karin Zamzow

data manager

Koordinierungszentr Klinische Studien,

Philipps-Universität

Marburg

Carmen

Schade-Brittinger

head of coordinating

centre for clinical trials

Medizinische

Biometrie und

Epidemiologie,

Philipps-Universität

Marburg

Hans-Helge Mueller

senior biostatistician

Correspondence to:

M Haake

m.haake@rheuma- ortho-zentrum.de

vacaine $1 \%$. Therapy was applied every two weeks plus or minus two days $(3 \times 4000$ impulses $)$. The head of the lithotripter was docked medially to the heel, and the ultrasound transducer from plantar to the insertion of the fascia. A cross hair on the monitor of the ultrasound guidance system constantly indicated the focus of the shock wave at the heel spur at the insertion of the fascia. The parameter positive energy flux density (ED+) is generally assumed to be the primary variable for physical and biological effects, and it was chosen to assure uniform treatment. ${ }^{15}$ The total positive dose was $0.96 \mathrm{~J} / \mathrm{mm}^{2}$, the energy flux density was $0.22 \mathrm{~mJ} / \mathrm{mm}^{2}$, and the positive pressure was 13.7 $\mathrm{MPa}$. The appropriate execution of the intervention was controlled for each centre.

Patients in the control group received the same regimen of placebo therapy under local anaesthesia. A polyethylene foil filled with air was fixed with ultrasound gel in front of the coupling cushion to reflect the shock waves. The set up in both groups was identical, and the sound created by the lithotripters was similar.

\section{Blinding}

Patients were blinded to their particular treatment allocation, and only the caregiver performing the intervention knew the treatment. Study doctors were not informed until assessment of the primary end point. The caregiver was not involved in follow up and was not allowed to decide about further treatment.

The clinical outcome was assessed by observers blinded to treatment allocation. The extent to which patients' remained blinded was assessed after the last intervention. Unblinding was possible after the assessment of the primary end point and only if the patient required further therapy.

\section{End points and side effects}

Follow up examinations were carried out at six and 12 weeks and at one year after the last intervention. The primary end point was the success rate after 12 weeks; success was defined by a Roles and Maudsley score of 1 or 2 and if the patient received no additional treatment. Additional treatment was allowed after assessment of the primary end point, and the amount was recorded. The modified Roles and Maudsley score is a patient administered scoring system (see table A on bmj.com). ${ }^{16}$ We used the German version, which is clinically relevant in plantar fasciitis and has been applied in major studies on extracorporeal shock wave therapy. $^{9}{ }^{10}$

Table 1 Characteristics of patients randomised to receive either extracorporeal shock wave therapy or placebo for chronic plantar fasciitis. Values are numbers (percentages) of patients unless stated otherwise

\begin{tabular}{lcc} 
Characteristic & Therapy group (n=135) & Placebo group (n=136) \\
\hline Sex: & & \\
\hline Male & $37(27)$ & $30(22)$ \\
\hline Female & $98(73)$ & $106(78)$ \\
\hline Foot affected: & $63(47)$ & $65(48)$ \\
\hline Right & $72(53)$ & $71(52)$ \\
\hline Left & $53.1(10.8)$ & $52.9(10.8)$ \\
\hline Mean (SD) age (years) & $29.4(4.9)$ & $29.7(4.8)$ \\
\hline Mean (SD) body mass index (kg/m²) & $13(10-24)$ & $13(9-24)$ \\
\hline Median (range) ${ }^{*}$ history of heel pain (months) & $12(8-18)$ & $11(8-21.5)$ \\
\hline Median (range) ${ }^{*}$ conservative treatment (months) & &
\end{tabular}

${ }^{*}$ First quarter to third quarter.
Secondary end points encompassed the Roles and Maudsley score and pain intensities (pain at rest, pain at night, pain at pressure, morning pain) on visual numeric rating scales ( 0 for no pain to 10 for unbearable pain), walking ability, and the need for additional treatments for one year after the last intervention. Side effects were noted.

\section{Statistics}

We carried out a two sided Fisher's exact test to compare the success rates at an overall significance level of $5 \%$. Patients were included according to the intention to treat principle. Absolute differences were calculated for the success rates, odds ratios, and exact 95\% confidence intervals. In addition we performed a stratified analysis by centre for verification of robustness of results. The secondary end points were analysed descriptively. An interim analysis was performed at $\alpha=0.005$ after examination of half the patients. The final analysis was performed at $\alpha=0.048$. The sample size of 272 patients was calculated with the aim of detecting a minimal clinically relevant difference of 20\% in the success rates (placebo: 35\%; extracorporeal shock wave therapy: $55 \%$ ) with a power of $80 \%$, allowing for a dropout rate of $20 \%$. Analyses were conducted with the validated programs SAS 8.2 and StatXact 5.0.

\section{Results}

Overall, 272 patients were randomised between March 1999 and February 2001 (figure). Personal characteristics were similar in both groups (table 1).

The required number of pulses and energy level for treatment was reached in all cases. Three patients in the placebo group accidentally received three sessions of extracorporeal shock wave therapy, and one patient in the therapy group received placebo. In the therapy group, one patient received no treatment because of a dorsal heel spur, one patient turned up for the first session only, and one patient missed the last session. One patient in the placebo group received no further intervention after the first session because of a deep vein thrombosis.

Blinding of the patients was successful: 95 (74\%) patients in the therapy group and $89(69 \%)$ patients in the placebo group thought they had been treated with extracorporeal shock wave therapy, the difference being less than $6 \%$.

\section{End points}

The primary end point could be assessed in 94\% of the patients (table 2). The difference in success rates was $3.6 \%(-8.0 \%$ to $15.1 \% ; \mathrm{P}=0.5927)$ and the odds ratio was 1.18 ( 0.675 to 2.07$)$. Despite two centres recruiting only nine and seven patients, none of the observed differences reached the minimal clinically relevant difference of $20 \%$. The odds ratio remained robust when data were stratified by centre $(1.20,0.674$ to 2.13$)$.

At the one year follow up, 91 of $113(81 \%)$ patients in the therapy group and 87 of $115(76 \%)$ in the placebo group had a Roles and Maudsley score of 1 or 2 (table 3). Additional treatment was sought by 41 $(36 \%)$ patients in the therapy group and $64(56 \%)$ patients in the placebo group. The number of conservative treatments was comparable between the groups except for the use of extracorporeal shock wave 
therapy (13 (12\%) patients in therapy group, 44 (38\%) patients in placebo group). One in each group had undergone surgery (see table B on bmj.com).

\section{Side effects}

Few side effects occurred during and after the treatment. More side effects were reported by the therapy group than by the placebo group $(24(18 \%) v$ $12(9 \%))$. Side effects were skin reddening $(16(12 \%)$ in therapy group; 5 (4\%) in placebo group), pain (7 (5\%) in therapy group; 2 (2\%) in placebo group), and local swelling $(3(2 \%)$ in therapy group; $0(0 \%)$ in placebo group). Less often reported were haematoma, nausea, dizziness, hair loss, and sleep disturbance. These were valued as non-serious effects, which in no case resulted in discontinuation of treatment. We expected a higher risk for side effects in the therapy group than in the placebo group (odds ratio 2.26, 1.02 to 5.18). We considered the case of deep vein thrombosis in the placebo group as not related to the treatment.

\section{Discussion}

We found no meaningful improvement of clinical outcome in patients treated with extracorporeal shock wave therapy for chronic plantar fasciitis compared with placebo, unlike previous studies. Although the success rates in patients with excellent or good results for the Roles and Maudsley score three months $(45.7 \%)$ and one year $(80.5 \%)$ after intervention were comparable to former trials, similar results could be achieved with placebo. ${ }^{10} 1217$

About three quarters of the patients in both groups had a good outcome one year after intervention. Reasons for the observed improvement could have been a spontaneous remission of plantar fasciitis, additional conservative treatment, or a sustained placebo effect. Despite this, we found no evidence of additional benefits from extracorporeal shock wave therapy.

Most of the newly reported trials on extracorporeal shock wave therapy for plantar fasciitis that were not included in a former systematic review ${ }^{14}$ also show deficiencies in the quality of the methods (for example, lack of a control group, ${ }^{18}{ }^{19}$ small sample size, ${ }^{180}$ unblinded design ${ }^{172021}$ ). Therefore these trials only provide limited evidence for the effectiveness of extracorporeal shock wave therapy.

We are aware of only three published randomised, blinded, placebo controlled trials. Two of them show benefits from extracorporeal shock wave therapy for plantar fasciitis. ${ }^{12}{ }^{13}$ However, the absolute difference of $17 \%$ in the first trial was not statistically significant and would not have met our definition of a clinically

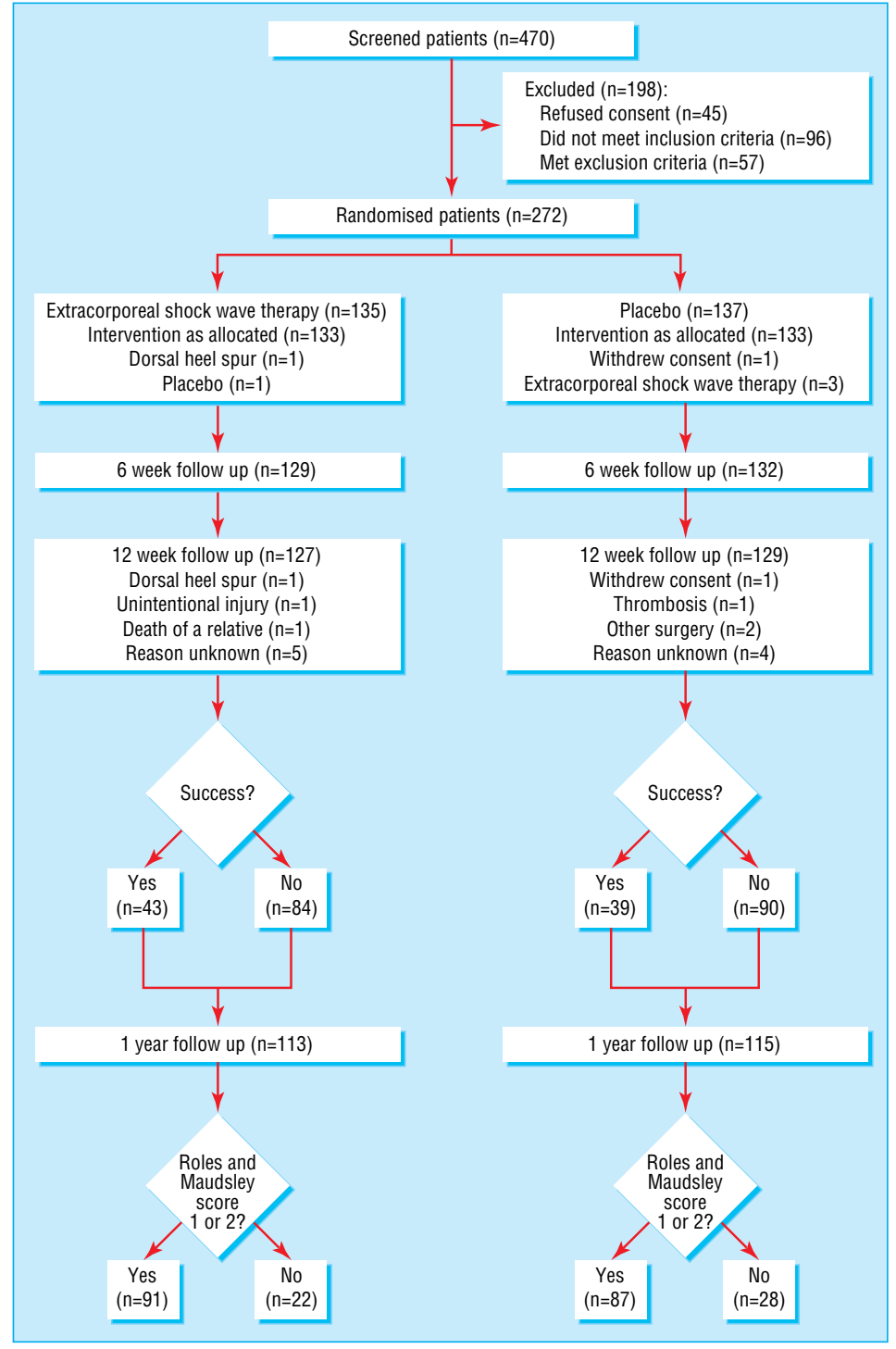

Flow of patients through trial

relevant result. ${ }^{12}$ The authors did not, however, report the method of randomisation. Different types of anaesthesia were used (foot block for extracorporeal shock wave therapy versus subcutaneous injection for placebo). Problems in the analysis and presentation of the data of this study have been published..$^{22}$ These factors may have influenced the results.

One study reported the alleviation of pain in the morning, ${ }^{13}$ but according to the Roles and Maudsely score, the American Orthopaedic Foot and Ankle

Table 2 Success rate of treatment for chronic plantar fasciitis six and 12 weeks and one year after extracorporeal shock wave therapy or placebo. Values are numbers (percentages) of patients unless stated otherwise

\begin{tabular}{|c|c|c|c|c|c|c|c|c|}
\hline Variable and group & Baseline & $\begin{array}{c}\text { Difference } \\
\text { (95\% CI) }\end{array}$ & 6 weeks & $\begin{array}{c}\text { Difference } \\
\text { (95\% CI) }\end{array}$ & 12 weeks & $\begin{array}{c}\text { Difference } \\
\text { (95\% CI) }\end{array}$ & 1 year & $\begin{array}{c}\text { Difference } \\
(95 \% \mathrm{CI})\end{array}$ \\
\hline \multicolumn{9}{|l|}{ Success rate: } \\
\hline Therapy & - & \multirow{2}{*}{ - } & - & & $43 / 127(34)$ & \multirow{2}{*}{3.6 (-8.0 to 15.1$)$} & - & \\
\hline Placebo & - & & - & & $39 / 129(30)$ & & - & \\
\hline \multicolumn{9}{|c|}{ Roles and Maudsley* score 1 or 2: } \\
\hline Therapy & $0 / 135(0)$ & \multirow{2}{*}{$\begin{array}{l}\text { Inclusion } \\
\text { criterion† }\end{array}$} & $28 / 129(22)$ & \multirow{2}{*}{$3.3(-13.7$ to 7.1$)$} & $58 / 127(46)$ & \multirow{2}{*}{$5.4(-7.0$ to 17.5$)$} & $91 / 113(81)$ & \multirow{2}{*}{$4.9(-6.0$ to 16.0$)$} \\
\hline Placebo & $0 / 136(0)$ & & $33 / 132(25)$ & & $52 / 129(40)$ & & $87 / 115(76)$ & \\
\hline
\end{tabular}

*Pain score according to Roles and Maudsley. ${ }^{18}$

tOnly patients with score of 3 or 4 included, therefore no patient had 1 or 2 at baseline. 
Table 3 Intensity of pain from chronic plantar fasciitis six and 12 weeks and one year after extracorporeal shock wave therapy or placebo

\begin{tabular}{|c|c|c|c|c|c|c|c|c|}
\hline \multirow[b]{2}{*}{ Variable } & \multicolumn{2}{|c|}{ Baseline } & \multicolumn{2}{|c|}{6 weeks } & \multicolumn{2}{|c|}{12 weeks } & \multicolumn{2}{|c|}{1 year } \\
\hline & $\begin{array}{c}\text { No of } \\
\text { patients }\end{array}$ & Mean (SD) & $\begin{array}{c}\text { No of } \\
\text { patients }\end{array}$ & Mean (SD) & $\begin{array}{c}\text { No of } \\
\text { patients }\end{array}$ & Mean (SD) & $\begin{array}{c}\text { No of } \\
\text { patients }\end{array}$ & Mean (SD) \\
\hline \multicolumn{9}{|c|}{ Pain at rest ${ }^{*}$ : } \\
\hline Therapy & 135 & $3.9(2.5)$ & 129 & $3.1(2.4)$ & 127 & $2.4(2.6)$ & 112 & $0.9(1.9)$ \\
\hline Placebo & 136 & $3.7(2.3)$ & 131 & $2.7(2.5)$ & 129 & $2.4(2.5)$ & 115 & $0.9(1.6)$ \\
\hline \multicolumn{9}{|c|}{ Pain at night*: } \\
\hline Therapy & 135 & $3.0(2.8)$ & 129 & $2.1(2.5)$ & 127 & $1.5(2.4)$ & 112 & $0.8(1.7)$ \\
\hline Placebo & 136 & $2.8(2.8)$ & 131 & $2.0(2.6)$ & 129 & $1.8(2.5)$ & 115 & $0.7(1.5)$ \\
\hline \multicolumn{9}{|c|}{ Pain at pressure*: } \\
\hline Therapy & 133 & $7.0(2.6)$ & 129 & $4.7(2.9)$ & 126 & $4.0(3.2)$ & 111 & $1.7(2.4)$ \\
\hline Placebo & 134 & $7.3(2.2)$ & 131 & $4.3(2.6)$ & 129 & $4.3(3.2)$ & 115 & $1.8(2.4)$ \\
\hline \multicolumn{9}{|c|}{ Morning pain*: } \\
\hline Therapy & 135 & $7.8(2.4)$ & 129 & $5.2(3.1)$ & 127 & $4.0(3.2)$ & 112 & $1.5(2.6)$ \\
\hline Placebo & 136 & $7.7(2.3)$ & 131 & $4.9(3.1)$ & 129 & $4.5(3.4)$ & 114 & $1.7(2.4)$ \\
\hline \multicolumn{9}{|c|}{ Walking ability†: } \\
\hline \multirow[t]{2}{*}{ Therapy } & 133 & $22.1(36.1)$ & 114 & $45.4(59.1)$ & 99 & $68.5(87.1)$ & 51 & $131.0(95.5)$ \\
\hline & 1 & Unlimited & 15 & Unlimited & 28 & Unlimited & 62 & Unlimited \\
\hline \multirow[t]{2}{*}{ Placebo } & 134 & $25.7(46.2)$ & 116 & $50.4(65.0)$ & 98 & 53.4 (77.3) & 53 & $115.1(102.5)$ \\
\hline & 2 & Unlimited & 16 & Unlimited & 31 & Unlimited & 62 & Unlimited \\
\hline
\end{tabular}

*11 point Likert scale.

†Minutes of pain free walking.

Society score, and pain scores, the findings were negative and comparable to our results. We do not believe that the only positive variable in that trial is a clinically relevant finding.

The negative findings of our study support the conclusion of the recently published third trial, although the treatment protocols of the studies differ slightly (mean total dose $1.4 \mathrm{~J} / \mathrm{mm}^{2}$ ). ${ }^{23}$ In contrast to our study the authors applied a minimal shock wave dose without anaesthesia $(3 \times 100$ impulses; 0.02 $\mathrm{mJ} / \mathrm{mm}^{2}$ ) instead of a sham therapy in the control group. This may have minimised a clinically relevant effect between both treatment groups.

Our results are only valid for therapeutic variables applied. This might not be a limitation, as the variables we tested reflect the true setting of extracorporeal shock wave therapy. The total energy of shock waves was higher in our trial than in most of the previous studies. ${ }^{9}{ }^{10}{ }^{17}$ Despite this, the use of different treatment variables might lead to different overall results, but the evidence for this could only be obtained from clinical trials with adequate study designs. We cannot recommend specific applications of extracorporeal shock wave therapy to be tested in further clinical studies because all major trials, using different shockwave variables and types of lithotripters, showed negative results.

We thank I R König, H Wolf, C Wöhner, O Maier-Boerries, and $\mathrm{C}$ Riemert who helped with the conduct of the trial or recruited patients, our colleagues in the participating centres without whom this study could not have been carried out, and the participants.

Contributors: $\mathrm{MH}$ was the principal investigator and will act as guarantor for the paper. $\mathrm{MB}, \mathrm{MH}$, and $\mathrm{HHM}$ designed the study. HHM was the biostatistician. CSB coordinated the study. HHM and KZ analysed the data. MH, HHM, CSB, and KZ prepared and finalised the paper. All authors interpreted the results, commented on the first draft, and approved the final version.

Funding: This trial was supported by the Deutsche Forschungsgemeinschaft (grant No 1079/2-1), the German Association for Orthopaedics and Orthopaedic Surgery, and the Association for Promoting Science and Research at the Rehberg Clinic, Germany. Dornier Medizintechnik Germany provided us with the shock wave equipment. They had no involvement in, or control over, the conduct of the study or the content of this paper.

\section{What is already known on this topic}

Observational trials recommend extracorporeal shock wave therapy as treatment for recalcitrant chronic plantar fasciitis

No evidence exists of its efficacy from well designed randomised clinical trials

\section{What this study adds}

Extracorporeal shock wave therapy is ineffective in the treatment of chronic plantar fasciitis

No clinically relevant difference was found in success rates between therapy and placebo after 12 weeks and a year

Three quarters of patients improved 12 months after intervention, irrespective of treatment

\section{Competing interests: None declared.}

Ethical approval: The study protocol was approved by the local ethics committees of the principal investigator (approval No $83 / 98$ ) and the participating centres.

De Maio M, Paine R, Mangine RE, Drez D. Plantar fasciitis. Orthopedics 1993;16:1153-63.

2 Kuhns JC. Changes in elastic adipose tissue. J Bone Joint Surg (Am) 1949;31:541-8.

3 Gill LH. Plantar fasciitis: diagnosis and conservative management. J Am Acad Orthop Surg 1997;5:109-17.

Davis PF, Severud E, Baxter DE. Painful heel syndrome: results of nonoperative treatment. Foot Ankle Int 1994;15:531-5.

5 Schepsis AA, Leach RE, Gorzyca J. Plantar fasciitis. Etiology, treatment, surgical results, and review of the literature Clin Orthop 1991:966:185-96.

Crawford F, Atkins D, Edwards J. Interventions for treating plantar heel pain. Cochrane Database Syst Rev 2000;3:CD000416.

7 Chaussy C, Eisenberger F, Wanner K. The use of shock waves for the destruction of renal calculi without direct contact Urol Res destruction of

8 Dahmen GP, Meiss L, Nam VC, Skruodies B. Extrakorporale Stosswellentherapie (ESWT) im knochennahen Weichteilbereich an der Schulter. Extracta Orthopaedica 1992;11:25-7.

9 Rompe JD, Hopf C, Nafe B, Bürger R. Low-energy extracorporeal shock wave therapy for painful heel: a prospective controlled single-blind study. Arch Orthop Trauma Surg 1996;115:75-9.

10 Rompe JD, Küllmer K, Riehle MH, Herbsthofer B, Eckardt A, Bürger R, et al. Effectiveness of low energy extracorporeal shock waves for chronic plantar fasciitis. Foot Ankle Surg 1996;2:215-21.

11 Krischek O, Rompe JD Herbsthofer B, Nafe B. Symptomatic low-eneroy shockwave therapy in heel pain and radiologically detected plantar heel spur. Z Orthop Ihre Grenzgeb 1998;136:169-74. 
12 Ogden JA, Alvarez R, Levitt R, Cross GL, Marlow M. Shock wave therapy for chronic proximal plantar fasciitis. Clin Orthop 2001;387:47-59.

13 Buch M, Knorr U, Fleming L, Theodore G, Amendola A, Bachmann C, et al. Extracorporeal shock wave therapy in plantar fasciitis: a review.

14 Böddeker IR, Schäfer H, Haake M. Extracorporeal shock-wave therapy (ESWT) in the treatment of plantar fasciitis-a biometrical review. Clin Rheumatol 2001;20:324-30.

15 Granz B, Kohler G. What makes a shock wave efficient in lithotripsy? J Stone Dis 1992;4:123-8.

16 Roles NC, Maudsley RH. Radial tunnel syndrome: resistant tennis elbow as a nerve entrapment. J Bone Joint Surg [Br] 1972;54:499-508.

17 Rompe JD, Schoellner C, Nafe B. Evaluation of low-energy extracorporeal shock-wave application for treatment of chronic plantar fasciitis. $J$ eal shock-wave application for trea
Bone Joint Surg [Am] 2002;84:335-41.

18 Alvarez R. Preliminary results on the safety and efficacy of the OssaTron Alvarez R. Preliminary results on the safety and efficacy of the Ossa
for treatment of plantar fasciitis. Foot Ankle Int 2002;23:197-203.
19 Wang CJ, Chen HS, Chen WS, Chen LM. Treatment of painful heels using extracorporeal shock wave. J Formos Med Assoc 2000;99:580-3.

20 Hammer DS, Rupp S, Kreutz A, Pape D, Kohn D, Seil R. Extracorporeal shockwave therapy (ESWT) in patients with chronic proximal plantar fasciitis. Foot Ankle Int 2002;23:309-13.

21 Weil LS Jr, Roukis TS, Weil LS, Borrelli AH. Extracorporeal shock wave therapy for the treatment of chronic plantar fasciitis: indications, protocol, intermediate results, and a comparison of results to fasciotomy. J Foot Ankle Surg 2002;41:166-72.

22 Beckman KD, Letter to the editor. Clin Orthop 2002;398:267-8.

23 Buchbinder R, Ptasznik R, Gordon J, Buchanan J, Prabaharan V, Forbes A. Ultrasound-guided extracorporeal shock wave therapy for plantar fasciitis: a randomized controlled trial. JAMA 2002;288:1364-72.

(Accepted 15 April 2003) 